\title{
BIFURCATION AND HAUSDORFF DIMENSION IN FAMILIES OF CHAOTICALLY DRIVEN MAPS WITH MULTIPLICATIVE FORCING
}

\author{
GERHARD KELLER AND ATSUYA OTANI
}

\begin{abstract}
We study bifurcations of invariant graphs in skew product dynamical systems driven by hyperbolic surface maps $T$ like Anosov surface diffeomorphisms or baker maps and with one-dimensional concave fibre maps under multiplicative forcing when the forcing is scaled by a parameter $r=e^{-t}$. For a range of parameters two invariant graphs (a trivial and a non-trivial one) coexist, and we use thermodynamic formalism to characterize the parameter dependence of the Hausdorff and packing dimension of the set of points where both graphs coincide. As a corollary we characterize the parameter dependence of the dimension of the global attractor $\mathcal{A}_{t}$ : Hausdorff and packing dimension have a common value $\operatorname{dim}\left(\mathcal{A}_{t}\right)$, and there is a critical parameter $\gamma_{c}^{-}$determined by the SRB measure of $T^{-1}$ such that $\operatorname{dim}\left(\mathcal{A}_{t}\right)=3$ for $t \leqslant \gamma_{c}^{-}$and $t \mapsto \operatorname{dim}\left(\mathcal{A}_{t}\right)$ is strictly decreasing for $t \in\left[\gamma_{c}^{-}, \gamma_{\max }\right)$.
\end{abstract}

\section{The General Setting AND a Review of main REsults}

In this paper we study bifurcations in skew product dynamical systems driven by a basis dynamical system $(\Theta, \mathcal{B}, T)$, where $(\Theta, \mathcal{B})$ is a measurable space and $T: \Theta \rightarrow \Theta$ a bi-measurable map. We denote the set of $T$-invariant probability measures and its subset of ergodic measures by $\mathcal{P}_{T}(\Theta)$ and $\mathcal{E}_{T}(\Theta)$, respectively. For the sake of simplicity, we will use the notation $\mu(\psi):=\int_{\Theta} \psi d \mu$ for $\mu \in \mathcal{P}_{T}(\Theta)$ and $\psi \in C(\Theta ; \mathbb{R})$. We also denote $\mathbb{R}_{\geqslant}:=[0, \infty)$.

1.1. The skew product system. For each parameter $t \in \mathbb{R}$ we define a skew-product transformation

$$
T_{t}: \Theta \times \mathbb{R}_{\geqslant} \rightarrow \Theta \times \mathbb{R}_{\geqslant}, \quad T_{t}(\theta, x):=\left(T \theta, f_{t}(\theta, x)\right),
$$

with a fibre function

$$
f_{t}: \Theta \times \mathbb{R}_{\geqslant} \rightarrow \mathbb{R}_{\geqslant}, \quad f_{t}(\theta, x):=e^{-t} g(\theta) h(x)
$$

where

$\triangleright h \in C^{1}\left(\mathbb{R}_{\geqslant} ; \mathbb{R}\right)$ is strictly concave with $h(0)=0, h^{\prime}(x)>0$ for $x>0, h^{\prime}(0)=1$, and $\lim _{x \rightarrow \infty} \frac{h(x)}{x}=0$,

$\triangleright g: \Theta \rightarrow(0, \infty)$ is bounded and measurable.

For $n \geqslant 2$ we define iteratively $f_{t}^{n}: \Theta \times \mathbb{R}_{\geqslant} \rightarrow \mathbb{R}_{\geqslant}$and $T_{t}: \Theta \times \mathbb{R}_{\geqslant} \rightarrow \Theta \times \mathbb{R}_{\geqslant}$,

$$
f_{t}^{n}(\theta, x):=f_{t}\left(T^{n-1} \theta, f_{t}^{n-1}(\theta, x)\right) \quad \text { and } \quad T_{t}^{n}(\theta, x):=T_{t}\left(T_{t}^{n-1}(\theta, x)\right) .
$$

Remark 1. The following properties are easily verified:

Date: September 24, 2021.

2010 Mathematics Subject Classification. 37D20, 37D35, 37G35, 37H20.

Key words and phrases. Skew product, global attractor, strange invariant graph, bifurcation, Hausdorff dimension.

This work is funded by DFG grant Ke 514/8-1. The authors thank B. Saussol and J. Schmeling for valuable help on some questions concerning the multifractal analysis of Birkhoff averages. 
a) $T_{t}^{n}(\theta, x)=\left(T^{n} \theta, f_{t}^{n}(\theta, x)\right)$ for $(\theta, x) \in \Theta \times \mathbb{R}_{\geqslant}$and $n \in \mathbb{N}_{0}$.

b) $\frac{d}{d x} f_{t}^{n}(\theta, x)>0$ and $\frac{d^{2}}{d x^{2}} f_{t}^{n}(\theta, x)<0$ for all $(\theta, x) \in \Theta \times(0, \infty)$ and $n \in \mathbb{N}$.

c) For each $t \in \mathbb{R}$ there is $M_{t}>0$ such that $f_{t}\left(\theta, M_{t}\right)<M_{t}$ for all $\theta \in \Theta$.

1.2. The maximal invariant function $\varphi_{t}$ and its zero set $N_{t}$. A function $\varphi: \Theta \rightarrow \mathbb{R}$ is invariant (or more precisely $T_{t}$-invariant), if

$$
T_{t}(\theta, \varphi(\theta))=(T \theta, \varphi(T \theta)) \quad \text { or, equivalently, } \quad f_{t}(\theta, \varphi(\theta))=\varphi(T \theta),
$$

for all $\theta \in \Theta$. The function $\varphi \equiv 0$ is always invariant. We call its graph $\Phi_{\text {base }}=\{(\theta, 0): \theta \in \Theta\}$ the baseline of the skew product system.

Since our fibre maps are monotone and strictly concave, this skew-product system possesses at most two essentially different measurable invariant functions, among them the maximal one, as the following lemma shows.

Lemma 1. a) For $t \in \mathbb{R}$ the maximal $T_{t}$-invariant function $\varphi_{t}: \Theta \rightarrow \mathbb{R}_{\geqslant}$,

$$
\varphi_{t}(\theta):=\lim _{n \rightarrow \infty} \psi_{t, n}(\theta)=\inf _{n} \psi_{t, n}(\theta),
$$

is well defined where $\psi_{t, n}(\theta):=f_{t}^{n}\left(T^{-n} \theta, M_{t}\right)$. It is indeed maximal, i.e. for every $T_{t^{-}}$ invariant function $\varphi$ we have that $0 \leqslant \varphi(\theta) \leqslant \varphi_{t}(\theta)$ for all $\theta \in \Theta$. Its graph is denoted by $\Phi_{t}:=\left\{\left(\theta, \varphi_{t}(\theta)\right): \theta \in \Theta\right\}$.

b) Let $\varphi$ be a measurable $T_{t}$-invariant function. Then we have for every $\mu \in \mathcal{E}_{T}(\Theta)$

$$
\varphi=0 \mu \text {-a.e. or } \varphi=\varphi_{t} \mu \text {-a.e. }
$$

The proof of part a) of this lemma is identical to the one in [5, pp.144-145], while part b) is contained in [5, Lemma 1]. Observe that in that reference the base system is an irrational rotation on $\mathbb{T}^{1}$, but only the invertibility and the ergodicity of the invariant (Lebesgue) measure are used for the proofs.

Depending on the stability properties of the fibre maps at $x=0$ relative to a measure $\mu \in \mathcal{E}_{T}(\Theta)$, the maximal invariant function $\varphi_{t}$ may be identical to zero, strictly positive, or and this is the most interesting case - it may have zeros without being identical to zero.

In this note we describe the measure theoretical and topological properties of the sets

$$
N_{t}:=\left\{\theta \in \Theta: \varphi_{t}(\theta)=0\right\},
$$

quantify the size of these sets in terms of their dimension and study the dependence of the dimension on the parameter $t$.

Remark 2. The following properties are immediate consequences of the definitions.

a) $\varphi_{t}$ is measurable. In particular, $N_{t} \in \mathcal{B}$.

b) $N_{t}$ is invariant under $T$, i.e. $T\left(N_{t}\right)=N_{t}=T^{-1}\left(N_{t}\right)$.

c) For $t<s$ we have $\varphi_{t}(\theta) \geqslant \varphi_{s}(\theta)$ for all $\theta \in \Theta$, whence $N_{t} \subseteq N_{s}$. We say therefore that the family $\left(N_{t}\right)_{t \in \mathbb{R}}$ is a filtration.

Remark 3. The set $\mathcal{A}_{t}:=\left\{(\theta, x): 0 \leqslant x \leqslant \varphi_{t}(\theta)\right\}$ is the global attractor of the map $T_{t}$. It is bounded from above by the upper bounding graph $\Phi_{t}$. As each ergodic $T_{t}$-invariant probability measure is supported by an invariant graph [1, Theorem 1.8.4(iv)], Lemma 1] shows that it is supported by $\Phi_{t}$ or by the baseline $\Phi_{\text {base }}$.

In view of the filtration property of the sets $\left(N_{t}\right)_{t \in \mathbb{R}}$ it is natural to define, for each $\theta \in \Theta$, a critical parameter $t_{c}(\theta)$ by

$$
t_{c}(\theta):=\inf \left\{t \in \mathbb{R}: \varphi_{t}(\theta)=0\right\} .
$$


Because of Remark 评 we have $t_{c}(\theta)=\sup \left\{t \in \mathbb{R}: \varphi_{t}(\theta)>0\right\}$, and for each $t \in \mathbb{R}$

$$
S_{t}:=\left\{\theta \in \Theta: t_{c}(\theta)=t\right\}=\bigcap_{t^{\prime \prime}>t} N_{t^{\prime \prime}} \backslash \bigcup_{t^{\prime}<t} N_{t^{\prime}} .
$$

As the baseline itself is a trivial invariant graph, these points can be understood as bifurcation points of invariant graphs.

1.3. The plan of this note. In section 2.1 we characterize the sets $N_{t}$ and $S_{t}$ in terms of Birkhoff averages

$$
\Gamma(\theta):=\liminf _{n \rightarrow \infty} \frac{1}{n} \sum_{k=1}^{n} \log g\left(T^{-k} \theta\right)
$$

which are closely related to the system's fibre-wise lower backwards Lyapunov exponents at the baseline. The main results are:

$\triangleright\{\Gamma<t\} \subseteq N_{t} \subseteq\{\Gamma \leqslant t\}$ and $S_{t}=\{\Gamma=t\}$.

$\triangleright \mu\left(S_{t} \backslash N_{t}\right)=0$ for each $\mu \in \mathcal{P}_{T}(\Theta)$.

In section 2.2 we characterize the same sets for each $\mu \in \mathcal{E}_{T}(\Theta)$ in terms of the averaged quantity

$$
\gamma(\mu):=\int \log g d \mu .
$$

By Birkhoff's ergodic theorem, $\gamma(\mu)=\Gamma(\theta)$ for $\mu$-a.e. $\theta$. The main observations are

$\triangleright \mu\left(N_{t}\right)=1$ if and only if $\gamma(\mu) \leqslant t$ and

$\triangleright \mu\left(S_{t}\right)=1$ if and only if $\gamma(\mu)=t$.

Finally, in section 3, we determine the Hausdorff dimensions $\operatorname{dim}_{H}$ and packing dimensions $\operatorname{dim}_{P}$ of the sets $N_{t}$ and $S_{t}$ for topologically mixing Anosov surface diffeomorphisms and baker maps using thermodynamic formalism. Define

$$
\gamma_{\min }:=\inf _{\mu \in \mathcal{P}_{T}(\Theta)} \gamma(\mu) \quad \text { and } \quad \gamma_{\max }:=\sup _{\mu \in \mathcal{P}_{T}(\Theta)} \gamma(\mu)
$$

In the Anosov case the main result reads: suppose $\gamma_{\min }<\gamma_{\max }$ and denote by $\gamma_{c}^{-}:=\gamma\left(\mu_{\mathrm{SRB}}^{-}\right)$ the average exponent of the SRB meaure of $T^{-1}$. There is a real analytic function $D$ : $\left(\gamma_{\min }, \gamma_{\max }\right) \rightarrow[0,1]$ such that $D\left(\gamma_{c}^{-}\right)=1, D^{\prime \prime}\left(\gamma_{c}^{-}\right)<0$,

$$
D^{\prime}(t)=\left\{\begin{array}{ll}
>0 & \text { for } t \in\left(\gamma_{\min }, \gamma_{c}^{-}\right) \\
<0 & \text { for } t \in\left(\gamma_{c}^{-}, \gamma_{\max }\right)
\end{array}, \quad\right. \text { and }
$$

$\triangleright \operatorname{dim}_{H}\left(N_{t}\right)=\operatorname{dim}_{H}\left(S_{t}\right)=D(t)+1$ for $t \in\left(\gamma_{\min }, \gamma_{c}^{-}\right)$,

$\triangleright \operatorname{dim}_{H}\left(\Theta \backslash N_{t}\right)=\operatorname{dim}_{H}\left(S_{t}\right)=\operatorname{dim}_{P}\left(\Theta \backslash N_{t}\right)=D(t)+1$ for $t \in\left(\gamma_{c}^{-}, \gamma_{\max }\right)$, and

$\triangleright \operatorname{dim}_{P}\left(N_{t}\right)=2>\operatorname{dim}_{H}\left(N_{t}\right)$ for $t \in\left(\gamma_{\min }, \gamma_{c}^{-}\right)$.

A number of proofs are deferred to section 4 .

\section{Characterization of the Sets $N_{t}$ And $S_{t}$ in terms of Lyapunov exponents}

2.1. The sets $N_{t}$ and $S_{t}$ via fibre-wise Lyapunov exponents. Recall that $\varphi_{t}$ is defined in (1) as a pullback limit. Therefore it is natural to characterize its zeros in terms of the fibre-wise lower backwards Lyapunov exponents at the baseline

$$
\Gamma_{t}(\theta):=\liminf _{n \rightarrow \infty} \frac{1}{n} \log \left|\frac{d}{d x} f_{t}^{n}\left(T^{-n} \theta, x\right)_{\mid x=0}\right|=\Gamma(\theta)-t .
$$


The following characterization of the set $S_{t}$ in terms of $\Gamma(\theta)$ is an essential point of this note. Under additional hyperbolicity assumptions it will be the key to a multifractal bifurcation analysis of the family $\left(\varphi_{t}\right)_{t \in \mathbb{R}}$.

Theorem 1 ( $N_{t}$ and $S_{t}$ via trajectory-wise Lyapunov exponents). Let $t \in \mathbb{R}$ and $\theta \in \Theta$.

a) If $\theta \notin N_{t}$, then $\Gamma(\theta) \geqslant t$, i.e. $\Gamma_{t}(\theta) \geqslant 0$.

b) If $\theta \in N_{t}$, then $\Gamma(\theta) \leqslant t$, i.e. $\Gamma_{t}(\theta) \leqslant 0$.

c) $\Gamma(\theta)=t_{c}(\theta)$ and $S_{t}=\{\theta \in \Theta: \Gamma(\theta)=t\}=\left\{\theta \in \Theta: \Gamma_{t}(\theta)=0\right\}$.

Although we have no proof, we do not believe that $S_{t} \subseteq N_{t}$. Instead we have the following characterization of points in $S_{t} \backslash N_{t}$.

Proposition 1 (Characterization of $\left.S_{t} \backslash N_{t}\right)$. Let $t \in \mathbb{R}$ and $\theta \in \Theta$. Then $\theta \in S_{t} \backslash N_{t}$ if and only if

$$
\sum_{k=1}^{\ell_{i}} \varphi_{t}\left(T^{-k} \theta\right)+\left|\log \varphi_{t}\left(T^{-\ell_{i}} \theta\right)\right|=o\left(\ell_{i}\right)
$$

along some subsequence $\left(\ell_{i}\right)_{i \geqslant 1}$.

The proofs of Theorem 1 and Proposition 1 are provided in section 4.1 .

Corollary 1. $\mu\left(S_{t} \backslash N_{t}\right)=0$ for each $\mu \in \mathcal{P}_{T}(\Theta)$ and $t \in \mathbb{R}$.

Proof. As $S_{t} \backslash N_{t}$ is $T$-invariant and as $0 \leqslant \varphi_{t} \leqslant M_{t}$, Proposition 1 implies

$$
\int_{S_{t} \backslash N_{t}} \varphi_{t} d \mu=\lim _{i \rightarrow \infty} \int_{S_{t} \backslash N_{t}} \frac{1}{\ell_{i}} \sum_{k=1}^{\ell_{i}} \varphi_{t} \circ T^{-k} d \mu=0,
$$

and as $\varphi_{t}>0$ on $S_{t} \backslash N_{t}$, it follows that $\mu\left(S_{t} \backslash N_{t}\right)=0$.

2.2. The sets $N_{t}$ and $S_{t}$ via average Lyapunov exponents. With respect to any invariant measure $\mu \in \mathcal{P}_{T}(\Theta)$ we define the average fibre-wise Lyapunov exponent at the baseline by

$$
\gamma_{t}(\mu):=\int \log \left|\frac{d}{d x} f_{t}(\cdot, x)_{\mid x=0}\right| d \mu=\gamma(\mu)-t .
$$

We note that

$$
\int \Gamma(\theta) d \mu(\theta)=\int \log g d \mu=\gamma(\mu)
$$

follows from Birkhoff's ergodic theorem, because $|\log g|$ is bounded by assumption.

Corollary $2\left(N_{t}\right.$ and $S_{t}$ via average Lyapunov exponents). For $\mu \in \mathcal{P}_{T}(\Theta)$ and $t \in \mathbb{R}$ we have:

a) If $\mu\left(N_{t}\right)=0$, then $\gamma_{t}(\mu)>0$, i.e. $\gamma(\mu)>t$.

b) If $\mu\left(N_{t}\right)=1$, then $\gamma_{t}(\mu) \leqslant 0$, i.e. $\gamma(\mu) \leqslant t$.

For ergodic $\mu$ both implications are equivalences and, furthermore,

c) $\mu\left(S_{t}\right)=1$ for $t=\gamma(\mu)$, and $\mu\left(S_{t}\right)=0$ otherwise.

Proof. a) If $\theta \in \Theta \backslash N_{t}$ for $\mu$-a.e. $\theta$, then $\Gamma_{t}(\theta) \geqslant 0$ for $\mu$-a.e. $\theta$ by Theorem 1 (1a), and as $\mu\left(S_{t}\right)=\mu\left(S_{t} \backslash N_{t}\right)=0$ by Corollary 1. $\Gamma_{t}(\theta)>0$ for $\mu$-a.e. $\theta$ by Theorem 1 1 c). Hence $\gamma_{t}(\mu)>0$. b) If $\theta \in N_{t}$ for $\mu$-a.e. $\theta$, then $\Gamma_{t}(\theta) \leqslant 0$ for $\mu$-a.e. $\theta$ by Theorem 1 (b), so that $\gamma_{t}(\mu) \leqslant 0$. 
If $\mu$ is ergodic, the cases a) and b) are exhaustive so that we have equivalences, and claim c) follows from Theorem $[\mathbb{1}$, since $\Gamma(\theta)=\gamma(\mu)$ for $\mu$ a.e. $\theta \in \Theta$ due to Birkhoff's ergodic theorem.

Let $\Gamma^{(n)}(\theta):=\frac{1}{n} \sum_{k=1}^{n} \log g\left(T^{-k} \theta\right)$ so that $\Gamma(\theta)=\liminf _{n \rightarrow \infty} \Gamma^{(n)}(\theta)$. Inspired by the representation of $S_{t}$ as $\{\theta \in \Theta: \Gamma(\theta)=t\}$ from Theorem 1] 1 we define the sets

$$
S_{t}^{\prime}:=\left\{\theta \in \Theta: \lim _{n \rightarrow \infty} \Gamma^{(n)}(\theta)=t\right\}
$$

and

$$
R_{t}^{\prime}:=\left\{\theta \in \Theta: \lim _{k \rightarrow \infty} \Gamma^{\left(n_{k}\right)}(\theta)=t \text { for some }\left(n_{k}\right)_{k} \subset \mathbb{N}\right\} .
$$

Observe that $S_{t}^{\prime} \subseteq S_{t} \subseteq R_{t}^{\prime}$. Both of these sets are very close to $S_{t}$ in the following measuretheoretic sense.

Corollary 3. For every $\mu \in \mathcal{E}_{T}(\Theta)$ and $t \in \mathbb{R}$ we have $\mu\left(S_{t} \backslash S_{t}^{\prime}\right)=0$ and $\mu\left(R_{t}^{\prime} \backslash S_{t}\right)=0$.

Proof. From Theorem 1 1 e), Corollary 邦 and Birkhoff's ergodic theorem we conclude that $\mu\left(S_{t}\right)=\mu\left(S_{t}^{\prime}\right)=\mu\left(R_{t}^{\prime}\right)=1$ for $t=\gamma(\mu)$ and that $\mu\left(S_{t}\right)=\mu\left(S_{t}^{\prime}\right)=\mu\left(R_{t}^{\prime}\right)=0$ for $t \neq \gamma(\mu)$. Thus, the differences are always sets of measure zero.

From now on let $\Theta$ be a compact metrizable space, $T: \Theta \rightarrow \Theta$ a homeomorphism, $g: \Theta \rightarrow$ $(0, \infty)$ continuous, and $\mathcal{B}$ the Borel $\sigma$-algebra on $\Theta$. Then $\varphi_{t}$ is upper-semi-continuous as an infimum of continuous functions. In particular, $N_{t}$ is a $G_{\delta}$-set.

Corollary 4. Under these topological assumptions:

a) $N_{t}=\emptyset$ for $t<\gamma_{\min }$.

b) $\emptyset \neq N_{t}$ and $N_{t} \neq \Theta$ for $\gamma_{\min } \leqslant t<\gamma_{\max }$.

c) $\mu\left(N_{\gamma_{\max }}\right)=1$ for all $\mu \in \mathcal{P}_{T}(\Theta)$.

d) $N_{t}=\Theta$ for $t>\gamma_{\max }$.

Proof. The semi-uniform ergodic theorem [10, Theorem 1.9] yields $\gamma_{\min } \leqslant \Gamma(\theta) \leqslant \gamma_{\max }$ for all $\theta \in \Theta$, whence a) and d) follow from Theorem 1. Corollary 2 shows that $N_{t}=\emptyset$ implies $t<\gamma(\mu)$ for each $\mu \in \mathcal{P}_{T}(\Theta)$, in particular $t<\gamma_{\min }$, and that $N_{t}=\Theta$ implies $t \geqslant \gamma_{\max }$. This shows assertion b). Finally, let $\mu \in \mathcal{P}_{T}(\Theta)$. As $\mu\left(S_{\gamma_{\max }} \backslash N_{\gamma_{\max }}\right)=0$ by Corollary 1 , we have $\mu\left(N_{\gamma_{\max }}\right)=\mu\left(N_{\gamma_{\max }} \cup S_{\gamma_{\max }}\right)=\mu\left(\bigcap_{t>\gamma_{\max }} N_{t}\right)=\mu(\Theta)=1$, where we used d) for the third identity. This proves c).

Remark 4. We do not know whether $N_{\gamma_{\max }}=\Theta$.

\section{Dimensions of the Sets $N_{t}$ And $S_{t}$ FOR HYPERBolic Systems}

Let $\Theta$ be a 2-dimensional compact Riemannian manifold, $T: \Theta \rightarrow \Theta$ a topologically mixing $C^{2}$-Anosov diffeomorphism, and $g: \Theta \rightarrow(0, \infty)$ a Hölder continuous function. Denote by $T_{\theta} \Theta=E^{s}(\theta) \oplus E^{u}(\theta)$ the splitting of the tangent fibre over $\theta \in \Theta$ into its stable and unstable subspaces, see [2, 3] for details. In the following, the Hausdorff dimension $\operatorname{dim}_{H}$ and the packing dimension $\operatorname{dim}_{P}$ are defined w.r.t. the associated Riemannian metric. We refer to [4] for their definitions.

Remark 5. As a lower backward ergodic average, $\Gamma: \Theta \rightarrow \mathbb{R}$ is constant along unstable manifolds. Therefore the sets $S_{t}$ and $S_{t}^{\prime}$ are unions of unstable manifolds, see Theorem 1 . 
The map $T^{-1}$ has a unique (and hence ergodic) Sinai-Ruelle-Bowen measure $\mu_{\mathrm{SRB}}^{-}$characterized by

$$
h_{T}\left(\mu_{\mathrm{SRB}}^{-}\right)+\mu_{\mathrm{SRB}}^{-}\left(\log \left\|d T \mid E^{s}\right\|\right)=\sup _{\mu \in \mathcal{P}_{T}(\Theta)}\left(h_{T}(\mu)+\mu\left(\log \left\|d T \mid E^{s}\right\|\right)\right)=0,
$$

see [3, section $4 \mathrm{~B}]$. We define the critical parameter $\gamma_{c}^{-}:=\gamma\left(\mu_{\mathrm{SRB}}^{-}\right)$. It is the "physical" or "observable" critical parameter of the family $\left(T_{t}\right)_{t \in \mathbb{R}}$ in the sense in which the SRB measure is often called a physical or observable measure, see Remark 7 for more details.

Theorem 2. Suppose that $\gamma_{\min }<\gamma_{\max }$ so that $\log g$ is not cohomologous to a constant. Then

$$
\begin{array}{ll}
\operatorname{dim}_{H}\left(N_{t}\right)=\operatorname{dim}_{H}\left(S_{t}\right)=D(t)+1 & \text { for } t \in\left(\gamma_{\min }, \gamma_{c}^{-}\right], \\
\operatorname{dim}_{H}\left(\Theta \backslash N_{t}\right)=\operatorname{dim}_{H}\left(S_{t}\right)=\operatorname{dim}_{P}\left(\Theta \backslash N_{t}\right)=D(t)+1 & \text { for } t \in\left[\gamma_{c}^{-}, \gamma_{\max }\right)
\end{array}
$$

with

$$
D(t):=\max \left\{\frac{h_{T}(\mu)}{\mu\left(-\log \left\|d T \mid E^{s}\right\|\right)}: \mu \in \mathcal{P}_{T}(\Theta) \text { and } \mu(\log g)=t\right\} .
$$

Furthermore, $D:\left(\gamma_{\min }, \gamma_{\max }\right) \rightarrow[0,1]$ is a real analytic function with $D\left(\gamma_{c}^{-}\right)=1, D^{\prime \prime}\left(\gamma_{c}^{-}\right)<$ 0 and

$$
D^{\prime}(t)=\left\{\begin{array}{ll}
>0 & \text { for } t \in\left(\gamma_{\min }, \gamma_{c}^{-}\right) \\
<0 & \text { for } t \in\left(\gamma_{c}^{-}, \gamma_{\max }\right)
\end{array} .\right.
$$

In addition, there is a unique equilibrium state $\mu_{t} \in \mathcal{E}_{T}(\Theta)$ such that $D(t)=\frac{h_{T}\left(\mu_{t}\right)}{\mu_{t}\left(-\log \left\|d T \mid E^{s}\right\|\right)}$, $\mu_{t}\left(S_{t}\right)=1$ and $\operatorname{dim}_{H}\left(\mu_{t}\right)=D(t)+1$.

Remark 6. In general one cannot expect that $D$ is concave on $\left(\gamma_{\min }, \gamma_{\max }\right)$, as is illustrated by [2, Proposition 9.2.2].

Corollary 5. $\operatorname{dim}_{P}\left(N_{t}\right)=2>\operatorname{dim}_{H}\left(N_{t}\right)$ for $t \in\left(\gamma_{\min }, \gamma_{c}^{-}\right)$.

Proof. As $T: \Theta \rightarrow \Theta$ is topologically transitive, the set $N_{t}$ is a dense $G_{\delta^{-}}$-set by Baire's category theorem, unless it is empty. Thus, $\operatorname{dim}_{P}\left(N_{t}\right)=2=D\left(\gamma_{c}^{-}\right)+1>\operatorname{dim}_{H}\left(N_{t}\right)$ for $t \in\left(\gamma_{\min }, \gamma_{c}^{-}\right)$.

Remark 7. The forward SRB measure $\mu_{\mathrm{SRB}}^{+}$, defined as in 16 but with $-\log \left\|d T \mid E^{u}\right\|$ instead of $\log \left\|d T \mid E^{s}\right\|$, defines another critical parameter $\gamma_{c}^{+}:=\gamma\left(\mu_{\mathrm{SRB}}^{+}\right)$. The forward and backward SRB measure coincide if and only if they are both absolutely continuous w.r.t. the volume measure [3, section $4 \mathrm{C}]$. Otherwise one can typically expect that $\gamma_{c}^{+} \neq \gamma_{c}^{-}$.

If $\gamma_{c}^{-}<t<\gamma_{c}^{+}$, then $\operatorname{dim}_{H}\left(\Theta \backslash N_{t}\right)=D(t)+1<2$. In particular, $N_{t} \subseteq \Theta$ has full volume such that the global attractor $\mathcal{A}_{t} \subseteq \Theta \times \mathbb{R} \geqslant$ has volume 0 . At the same time, the fibre-wise forward Lyapunov exponent at the base satisfies $\lim _{n \rightarrow \infty} \frac{1}{n} \log \left|\frac{d}{d x} f_{t}^{n}(\theta, x)_{\mid x=0}\right|=$ $\int \log \left|\frac{d}{d x} f_{t}(\theta, x)_{\mid x=0}\right| d \mu_{\mathrm{SRB}}^{+}=\gamma_{c}^{+}-t>0$ for a.e. $\theta \in \Theta$ w.r.t. the volume measure [3, section $4 \mathrm{C}]$.

If $\gamma_{c}^{+}<t<\gamma_{c}^{-}$, then $\operatorname{dim}_{H}\left(N_{t}\right)=D(t)+1<2$. In particular, $N_{t} \subseteq \Theta$ has zero volume. But now the fibre-wise forward Lyapunov exponent at the base is strictly negative so that the concavity of the fibre maps implies that $\lim _{n \rightarrow \infty} f_{t}^{n}(\theta, x)=0$ for a.e. $\theta \in \Theta$ w.r.t. the volume measure and all $x \in \mathbb{R}_{\geqslant}$.

Theorem 3. Suppose that $\gamma_{\min }<\gamma_{\max }$ so that $\log g$ is not cohomologous to a constant. Then

$$
\begin{array}{ll}
\operatorname{dim}_{H}\left(\mathcal{A}_{t}\right)=\operatorname{dim}_{P}\left(\mathcal{A}_{t}\right)=3 & \text { for } t \in\left(\gamma_{\min }, \gamma_{c}^{-}\right] \\
\operatorname{dim}_{H}\left(\mathcal{A}_{t}\right)=\operatorname{dim}_{P}\left(\mathcal{A}_{t}\right)=D(t)+2 & \text { for } t \in\left[\gamma_{c}^{-}, \gamma_{\max }\right) .
\end{array}
$$




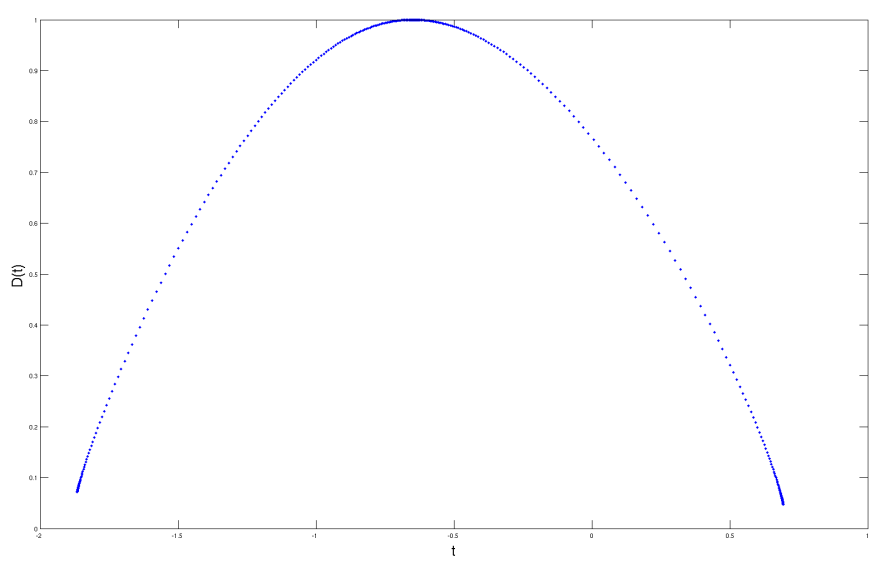

Figure 1. The function $D(t)$ for a baker map with $a=0.45$ and $g(u, v)=$ $1.001+\cos (2 \pi v)$.

Remark 8. Theorem 2 remains true also for Baker maps $B_{a}:[0,1]^{2} \rightarrow[0,1]^{2}$ for $a \in(0,1)$,

$$
B_{a}(u, v):=\left\{\begin{array}{ll}
\left(\frac{u}{a}, a v\right) & \text { for } u<a \\
\left(\frac{u-a}{1-a}, a+(1-a) v\right) & \text { for } u \geqslant a
\end{array} .\right.
$$

In this case $\mu_{\mathrm{SRB}}^{-}=\mu_{\mathrm{SRB}}^{+}=m^{2}$ (the Lebesgue measure on $[0,1)^{2}$ ) so that $\gamma_{c}^{-}=\gamma_{c}^{+}=$ $\int \log g d m^{2}$, and

$$
\log \left\|d B_{a} \mid E^{s}\right\|=\log \left(a \mathbb{1}_{[0, a) \times[0,1)}+(1-a) \mathbb{1}_{[a, 1) \times[0,1)}\right) .
$$

The reason that the same (indeed, even much simpler) arguments apply is essentially that the discontinuity line is the boundary of a Markov partition. So there are no additional difficulties passing from the symbolic to the (piecewise) smooth system, see also [9].

Remark 9. For a baker map with $a=0.45$ and for $g(u, v)=1.001+\cos (2 \pi v)$ we determined $D(t)$ numerically, see Figure 3 . The seeming discontinuities at $\gamma_{\min }$ and $\gamma_{\max }$ are numerical artefacts due to the fact that only trajectories of length $N=21$ were used to approximate the equilibrium states $\mu_{t}$.

In order to see that the limits of $D(t)$ for $t \rightarrow \gamma_{\min / \max }$ are indeed zero, it suffices to show that all $\mu \in \mathcal{P}_{T}(\Theta)$ which minimize or maximize $\mu(\log g)$ have entropy $h_{T}(\mu)=0$, because then $\lim _{t \rightarrow \gamma_{\min / \max }} h_{T}\left(\mu_{t}\right)=0$ due to the upper semicontinuity of $h_{T}$, and so also $\lim _{t \rightarrow \gamma_{\min } / \max } D(t)=0$, because the Lyapunov exponents of all $\mu_{t}$ are uniformly bounded away from zero. For $t \rightarrow \gamma_{\max }$ this yields a full proof, because it is easily seen that only the point masses in the fixed points $(0,0)$ and $(1,1)$ maximize $\mu(\log g)$, and so $\lim _{t \rightarrow \gamma_{\max }} D(t)=$ 0 . For $t \rightarrow \gamma_{\min }$ the situation is less clear. There is numerical evidence (no proof) that the equidistribution on a 3 -cycle minimizes $\mu(\log g)$. If this is indeed the case, then also $\lim _{t \rightarrow \gamma_{\min }} D(t)=0$. See also [7] for more details.

\section{Proofs}


4.1. Proofs from section 2, Let $\left(\psi_{t, n}\right)_{n \geqslant 0}$ and $M_{t}>0$ be as defined in Lemma 1, and denote $g_{t}:=e^{-t} g$.

Proof of Theorem 1. a) Let $\theta \in \Theta$ such that $\Gamma_{t}(\theta)<0$. There are $\delta>0$ and integers $n_{1}<n_{2}<\ldots$ such that

$$
\frac{d}{d x} f_{t}^{n_{j}}\left(T^{-n_{j}} \theta, x\right)_{\mid x=0}=\prod_{i=1}^{n_{j}} g_{t}\left(T^{-i} \theta\right) \leqslant e^{-\delta n_{j}}
$$

for all $j \in \mathbb{N}$. As $\frac{d^{2}}{d x^{2}} f_{t}^{n_{j}}\left(T^{-n_{j}} \theta, x\right)<0$ for all $x \in(0, \infty)$ (cf. Remark 1 ), we have

$$
\varphi_{t}(\theta) \leqslant \psi_{t, n_{j}}(\theta)=f_{t}^{n_{j}}\left(T^{-n_{j}} \theta, M_{t}\right) \leqslant e^{-\delta n_{j}} M_{t} \stackrel{j \rightarrow \infty}{\longrightarrow} 0 .
$$

b) Let $\theta \in \Theta$ such that $\Gamma_{t}(\theta)>0$. There are $\delta>0$ and $C>0$ such that, for all $n \in \mathbb{N}_{0}$,

$$
\prod_{i=1}^{n} g_{t}\left(T^{-i} \theta\right) \geqslant C e^{\delta n}
$$

For a contradiction, suppose $\varphi_{t}(\theta)=0$. Let $\varepsilon_{0}>0$ and $n_{0} \in \mathbb{N}$ such that

$$
\inf _{x \in\left(0, \varepsilon_{0}\right)} \frac{h(x)}{x} \geqslant e^{-\delta} \text { and } \psi_{t, n_{0}}(\theta)<\varepsilon_{0} .
$$

Let $k(n):=\max \left\{k \leqslant n: f_{t}^{k}\left(T^{-n} \theta, M_{t}\right) \geqslant \varepsilon_{0}\right\}$. As $k(n)<n$ if $n \geqslant n_{0}$, we have for $j \in\{0, \ldots, n-k(n)-1\}$, i.e. for $n-j \in\{k(n)+1, \ldots, n\}$,

$$
\begin{aligned}
f_{t}^{n-k(n)-j}\left(T^{-n+k(n)} \theta, \varepsilon_{0}\right) & \leqslant f_{t}^{n-k(n)-j}\left(T^{-n+k(n)} \theta, f_{t}^{k(n)}\left(T^{-n} \theta, M_{t}\right)\right) \\
& =f_{t}^{n-j}\left(T^{-n} \theta, M_{t}\right)<\varepsilon_{0} .
\end{aligned}
$$

Using now 222 with $j=0$ and then (21) and (22) repeatedly, we obtain for $n \geqslant n_{0}$

$$
\begin{aligned}
\psi_{t, n}(\theta) & =f_{t}^{n}\left(T^{-n} \theta, M_{t}\right) \\
& \geqslant f_{t}^{n-k(n)}\left(T^{-n+k(n)} \theta, \varepsilon_{0}\right) \\
& =g_{t}\left(T^{-1} \theta\right) h\left(f_{t}^{n-k(n)-1}\left(T^{-n+k(n)} \theta, \varepsilon_{0}\right)\right) \\
& \geqslant g_{t}\left(T^{-1} \theta\right) e^{-\delta} f_{t}^{n-k(n)-1}\left(T^{-n+k(n)} \theta, \varepsilon_{0}\right) \\
& =g_{t}\left(T^{-1} \theta\right) e^{-\delta} g_{t}\left(T^{-2} \theta\right) h\left(f_{t}^{n-k(n)-2}\left(T^{-n+k(n)} \theta, \varepsilon_{0}\right)\right) \\
& \geqslant g_{t}\left(T^{-1} \theta\right) e^{-\delta} g_{t}\left(T^{-2} \theta\right) e^{-\delta} f_{t}^{n-k(n)-2}\left(T^{-n+k(n)} \theta, \varepsilon_{0}\right) \\
& \vdots \\
& \geqslant\left(\prod_{i=1}^{n-k(n)} g_{t}\left(T^{-i} \theta\right)\right)\left(e^{-\delta}\right)^{n-k(n)} \varepsilon_{0} .
\end{aligned}
$$

With 20 and the first estimate of (21) we obtain from 23

$$
\psi_{t, n}(\theta) \geqslant C e^{\delta(n-k(n))} e^{-\delta(n-k(n))} \varepsilon_{0}=C \varepsilon_{0},
$$

and therefore $\varphi_{t}(\theta) \geqslant C \varepsilon_{0}$. This contradicts the assumption. 
c) This is a direct consequence of a and b].

Proof of Proposition 1 .

Let $\theta \in \Theta \backslash N_{t}$, i.e. $\varphi_{t}(\theta)>0$. By definition of the functions $\psi_{t, n}$ we have

$$
\psi_{t, n}(\theta)=g_{t}\left(T^{-1} \theta\right) h\left(\psi_{t, n-1}\left(T^{-1} \theta\right)\right)
$$

so that

$$
\log \psi_{t, n}(\theta)=\log g_{t}\left(T^{-1} \theta\right)+H\left(\psi_{t, n-1}\left(T^{-1} \theta\right)\right)+\log \psi_{t, n-1}\left(T^{-1} \theta\right)
$$

where $H(x):=\log \left(x^{-1} h(x)\right)$. As $h$ is concave and $h^{\prime}(0)=1, H$ extends to a decreasing function from $[0, \infty)$ to $(-\infty, 0]$ with $H(0)=0$ and $H^{\prime}(0)=\frac{1}{2} h^{\prime \prime}(0)<0$. Iterating this identity $\ell$ times $(1 \leqslant \ell \leqslant n)$ yields

$$
\log \psi_{t, n}(\theta)=\sum_{k=1}^{\ell} \log g_{t}\left(T^{-k} \theta\right)+\sum_{k=1}^{\ell} H\left(\psi_{t, n-k}\left(T^{-k} \theta\right)\right)+\log \psi_{t, n-\ell}\left(T^{-\ell} \theta\right) .
$$

For fixed $\ell \in \mathbb{N}$ we get in the limit $n \rightarrow \infty$

$$
\log \varphi_{t}(\theta)=\sum_{k=1}^{\ell} \log g_{t}\left(T^{-k} \theta\right)+\sum_{k=1}^{\ell} H\left(\varphi_{t}\left(T^{-k} \theta\right)\right)+\log \varphi_{t}\left(T^{-\ell} \theta\right) .
$$

Recall that $\theta \in \Theta \backslash N_{t}$. Because of Theorem 1 1 C $), \theta \in S_{t}$ if and only if $\sum_{k=1}^{\ell_{i}} \log g_{t}\left(T^{-k} \theta\right)=$ $o\left(\ell_{i}\right)$ along some subsequence $\left(\ell_{i}\right)_{i \in \mathbb{N}}$. In view of (26) this is equivalent to

$$
\sum_{k=1}^{\ell_{i}}\left|H\left(\varphi_{t}\left(T^{-k} \theta\right)\right)\right|+\left|\log \varphi_{t}\left(T^{-\ell_{i}} \theta\right)\right|=o\left(\ell_{i}\right) .
$$

As $H^{\prime}(0)<0$, there is a constant $C>0$ such that

$$
C^{-1} \varphi_{t}\left(T^{-k} \theta\right) \leqslant\left|H\left(\varphi_{t}\left(T^{-k} \theta\right)\right)\right| \leqslant C \varphi_{t}\left(T^{-k} \theta\right) \quad(k \geqslant 0),
$$

so that (27) is equivalent to

$$
\sum_{k=1}^{\ell_{i}} \varphi_{t}\left(T^{-k} \theta\right)+\left|\log \varphi_{t}\left(T^{-\ell_{i}} \theta\right)\right|=o\left(\ell_{i}\right)
$$

\subsection{Proof of Theorem 2,}

Remark 10. We refer to [3, 6, 8 ] for the following well-known facts about topologically mixing Anosov diffeomorphisms:

$\triangleright$ The entropy function $h_{T}(\cdot): \mathcal{P}_{T}(\Theta) \rightarrow \mathbb{R} \geqslant$ is upper semi-continuous, as $T$ is expansive.

$\triangleright$ For Hölder-continuous functions $\phi, \psi: \Theta \rightarrow \mathbb{R}$ the function $t \mapsto P(t \phi+\psi)$ is real analytic and strictly convex, unless $\phi$ is cohomologous to a constant, where $P(t \phi+\psi)$ denotes the topological pressure of $T$ for the potential $t \phi+\psi$.

We shall prepare three Lemmas, before we prove the theorem. In this section we assume that $\gamma_{\min }<\gamma_{\max }$. Recall that we defined $D(t)$ as

$$
D(t)=\max \left\{\frac{h_{T}(\mu)}{\mu\left(-\log \left\|d T \mid E^{s}\right\|\right)}: \mu \in \mathcal{P}_{T}(\Theta) \text { and } \gamma(\mu)=t\right\} .
$$


Lemma 2. $\operatorname{dim}_{H}\left(S_{t}^{\prime}\right)=D(t)+1$ for $t \in\left(\gamma_{\min }, \gamma_{\max }\right)$. Furthermore, there is a real analytic function $q:\left(\gamma_{\min }, \gamma_{\max }\right) \rightarrow \mathbb{R}$ such that for the unique equilibrium measure $\mu_{t} \in \mathcal{E}_{T}(\Theta)$ of the potential $q(t) \log g_{t}-D(t) \log \left\|d T \mid E^{s}\right\|$ the following holds:

$$
\mu_{t}\left(S_{t}\right)=1, \quad D(t)=\frac{h_{T}\left(\mu_{t}\right)}{\mu_{t}\left(-\log \left\|d T \mid E^{s}\right\|\right)}, \quad \text { and } \quad \operatorname{dim}_{H}\left(\mu_{t}\right)=D(t)+1 .
$$

Proof. For $\theta \in \Theta$ denote by $V_{l o c}^{s}(\theta)$ the (suitably defined) local stable manifold of $T$ through $\theta$. Then [2, Theorem 12.2.2] asserts that $\operatorname{dim}_{H}\left(S_{t}^{\prime}\right)=\operatorname{dim}_{H}\left(S_{t}^{\prime} \cap V_{l o c}^{s}(\theta)\right)+1$ for each $\theta \in S_{t}^{\prime}$. With the same argument as in [2, Example 7.2.5] one shows that $\operatorname{dim}_{H}\left(S_{t}^{\prime} \cap V_{l o c}^{s}(\theta)\right)$ coincides with the $u$-dimension of $S_{t}^{\prime}$ for the function $u=-\log \left\|d T \mid E^{s}\right\|$, and [2, Theorem 10.1.4] asserts that this $u$-dimension takes the value $D(t)$. (For the application of this last theorem observe that all Hölder potentials have a unique equilibrium state.) The assertions on $q(t)$ and $\mu_{t}$ follow from [2, Theorems 10.1.4 and 10.3.1] together with Corollary 3, [2, Lemma 10.1.6] and its proof.

Lemma 3. $D:\left(\gamma_{\min }, \gamma_{\max }\right) \rightarrow[0,1]$ is a real analytic function such that $D\left(\gamma_{c}^{-}\right)=1$, $D^{\prime \prime}\left(\gamma_{c}^{-}\right)<0$ and

$$
D^{\prime}(t)=\left\{\begin{array}{ll}
>0 & \text { for } t \in\left(\gamma_{\min }, \gamma_{c}^{-}\right) \\
<0 & \text { for } t \in\left(\gamma_{c}^{-}, \gamma_{\max }\right)
\end{array} .\right.
$$

Proof of Lemma 3. For this proof, we will extend the proof of [2, Theorem 10.3.1]: Denote $(u, \Phi):=\left(-\log \left\|d T \mid E^{s}\right\|, \log g\right)$ and consider the real analytic function $Q(\delta, q, t):=$ $P(q(\Phi-t)-\delta u)$. In that proof it is shown that the equations

$$
\left\{\begin{array}{c}
Q(D(t), q(t), t)=0 \\
\frac{\partial Q}{\partial q}(D(t), q(t), t)=0
\end{array}\right.
$$

determine a real analytic function $(D, q):\left(\gamma_{\min }, \gamma_{\max }\right) \rightarrow \mathbb{R}^{2}$. These equations are further equivalent to

$$
\left\{\begin{array}{l}
h_{T}\left(\mu_{t}\right)+\mu_{t}(q(t) \Phi-D(t) u)=q(t) t \\
\mu_{t}(\Phi)=t
\end{array}\right.
$$

Differentiating the first equality of (32) with respect to $t$ and using the second one, we obtain

$$
D^{\prime}(t)=\frac{q(t)}{\frac{\partial Q}{\partial \delta}(D(t), q(t), t)} .
$$

Similarly, differentiating the second equality of (32) and using (34), we obtain

$$
q^{\prime}(t)=\frac{1}{\frac{\partial^{2} Q}{\partial q^{2}}(D(t), q(t), t)}\left(1-q(t) \frac{\frac{\partial^{2} Q}{\partial \delta \partial q}(D(t), q(t), t)}{\frac{\partial Q}{\partial \delta}(D(t), q(t), t)}\right) .
$$

From the second equality of (33) it follows $\mu_{t}\left(S_{t}^{\prime}\right)=1$ by Birkhoff's theorem, whence $\mu_{t}\left(S_{t}\right)=1$ in view of Corollary 3. Moreover, from (33) we obtain

$$
D(t)=\frac{h_{T}\left(\mu_{t}\right)}{\mu_{t}(u)} .
$$

Furthermore, from $(16)$ we have

$$
\left\{\begin{array}{l}
Q\left(1,0, \gamma_{c}^{-}\right)=P(-u)=h_{T}\left(\mu_{\mathrm{SRB}}^{-}\right)-\mu_{\mathrm{SRB}}^{-}(u)=0 \\
\frac{\partial Q}{\partial q}\left(1,0, \gamma_{c}^{-}\right)=\mu_{\mathrm{SRB}}^{-}(\varphi)-\gamma_{c}^{-}=0
\end{array}\right.
$$


so that

$$
\left(1,0, \gamma_{c}^{-}\right)=\left(D\left(\gamma_{c}^{-}\right), q\left(\gamma_{c}^{-}\right), \gamma_{c}^{-}\right)
$$

In view of (34), we have also

$$
D^{\prime}\left(\gamma_{c}^{-}\right)=0 .
$$

Finally, differentiating (34) we obtain

$$
D^{\prime \prime}(t)=\frac{q^{\prime}(t)}{\frac{\partial Q}{\partial \delta}}-q(t) \frac{D^{\prime}(t) \frac{\partial^{2} Q}{\partial \delta^{2}}+q^{\prime}(t) \frac{\partial^{2} Q}{\partial \delta \partial q}+\frac{\partial^{2} Q}{\partial \delta \partial t}}{\left(\frac{\partial Q}{\partial \delta}\right)^{2}}
$$

where all partial derivatives are evaluated at $(D(t), q(t), t)$. Observing that $\frac{\partial^{2} Q}{\partial \delta \partial t}=0$ and substituting (34) we obtain

$$
D^{\prime \prime}(t)=\frac{1}{\frac{\partial Q}{\partial \delta}}\left(q^{\prime}(t)-D^{\prime}(t)\left(D^{\prime}(t) \frac{\partial^{2} Q}{\partial \delta^{2}}+q^{\prime}(t) \frac{\partial^{2} Q}{\partial \delta \partial q}\right)\right)
$$

with all partial derivatives again evaluated at $(D(t), q(t), t)$. As we assume that $\gamma_{\min }<\gamma_{\max }$, the map $q \mapsto Q(\delta, q, t)$ is strictly convex (c.f. Remark 10). Hence, we obtain from (35) and (41) together with (38) and (39),

$$
q^{\prime}\left(\gamma_{c}^{-}\right)=\frac{1}{\frac{\partial^{2} Q}{\partial q^{2}}\left(1,0, \gamma_{c}^{-}\right)}>0 \quad \text { and } \quad D^{\prime \prime}\left(\gamma_{c}^{-}\right)=\frac{q^{\prime}\left(\gamma_{c}^{-}\right)}{\frac{\partial Q}{\partial \delta}\left(1,0, \gamma_{c}^{-}\right)}<0
$$

Now consider the curve $C:\left(\gamma_{\min }, \gamma_{\max }\right) \rightarrow[0,1] \times \mathbb{R}$ defined by $C(t)=(D(t), q(t))$. In view of (38) and the first inequality of (42) this curve runs across the point $(1,0)$ in $t=\gamma_{c}^{-}$ from left to right. As sign $\left(D^{\prime}(t)\right)=-\operatorname{sign}(q(t))$ by $(34), D^{\prime}(t)$ can change its sign only when $q(t)=0$, i.e. only if $t=\gamma_{c}^{-}$and hence $D(t)=1$. Therefore $D^{\prime}(t)>0$ for $t<\gamma_{c}^{-}$and $D^{\prime}(t)<0$ for $t>\gamma_{c}^{-}$. by

Recall that for $\nu \in \mathcal{P}(\Theta)$ the upper and lower point-wise dimensions at $\theta \in \Theta$ are defined

$$
\bar{d}_{\nu}(\theta):=\limsup _{r \rightarrow 0} \frac{\log \nu(B(\theta, r))}{\log r} \text { and } \quad \underline{d}_{\nu}(\theta):=\liminf _{r \rightarrow 0} \frac{\log \nu(B(\theta, r))}{\log r} .
$$

If $\bar{d}_{\nu}(\theta)=\underline{d}_{\nu}(\theta)$, we denote this with $d_{\nu}(\theta)$.

To formulate the next lemma we define the sets

$$
N_{t}^{\geqslant}:=\{\theta \in \Theta: \Gamma(\theta) \geqslant t\} \quad \text { and } \quad N_{t}^{\leqslant}:=\left\{\theta \in \Theta: \limsup _{n \rightarrow \infty} \Gamma^{(n)}(\theta) \leqslant t\right\} .
$$

Lemma 4. Let $t \in\left(\gamma_{\min }, \gamma_{\max }\right)$. There is $\nu_{t} \in \mathcal{P}(\Theta)$ (which is in general not T-invariant) such that $\nu_{t}\left(S_{t}^{\prime}\right)=1$ with the following properties:

(1) $d_{\nu_{t}}(\theta)=D(t)+1$ for $\nu_{t}$-a.e. $\theta \in \Theta$,

(2) $\bar{d}_{\nu_{t}}(\theta) \leqslant D(t)+1$ for each $\theta \in S_{t}^{\prime}$,

(3) $\underline{d}_{\nu_{t}}(\theta) \leqslant D(t)+1$ for each $\theta \in R_{t}^{\prime}$,

(4) $\bar{d}_{\nu_{t}}(\theta) \leqslant D(t)+1$ for each $\theta \in N_{t}^{\leqslant}$provided that $t<\gamma_{c}^{-}$, and

(5) $\bar{d}_{\nu_{t}}(\theta) \leqslant D(t)+1$ for each $\theta \in N_{t}^{\geqslant}$provided that $t>\gamma_{c}^{-}$. 
Proof of Lemma 4. We apply the very general result [2, Theorem 12.3.1] with slight modifications. Here is a dictionary between our notation and the notation from [2]:

\begin{tabular}{c|c} 
Notation from [2] & Our notation \\
\hline$\Lambda, f, \mathcal{M}$ & $\Theta, T, \mathcal{P}_{T}(\Theta)$ \\
$\kappa$ & 1 \\
$\mathcal{P}^{+}(\cdot), \mathcal{P}^{-}(\cdot)$ & $1, \gamma(\cdot)$ \\
$\Phi^{+}, \Phi^{-}, \Psi^{+}, \Psi^{-}$ & $1, \log g, 1,1$ \\
$\alpha, \beta$ & $1, t$ \\
$K_{\alpha}^{+}, K_{\beta}^{-}$ & $\Theta, S_{t}^{\prime}$ \\
$d^{+}, d^{-}$ & $1, D(t)$ \\
$q^{+}, q^{-}$ & $1, q(t)$
\end{tabular}

We note that the assumption $\alpha \in \operatorname{int} \mathcal{P}^{+}(\mathcal{M})$ of [2, Theorem 12.3.1] is not satisfied in our setting, as $\mathcal{P}^{+}(\mathcal{M})=\{1\}=\{\alpha\}$. This assumption is only used to assure the existence of $q^{+}$ with the properties claimed in [2, Lemma 12.3.3]; but these properties are trivially satisfied in our case.

Now we can conclude immediately from [2, Theorem 12.3.1] and Lemma 2 that

$$
\nu_{t}\left(S_{t}^{\prime}\right)=\nu_{t}\left(K_{\alpha}^{+} \cap K_{\beta}^{-}\right)=1 \text {, }
$$

and

$$
\begin{gathered}
d_{\nu_{t}}(\theta)=D(t)+1 \quad \text { for } \nu_{t} \text {-a.e. } \theta \in \Theta, \\
\bar{d}_{\nu_{t}}(\theta) \leqslant D(t)+1 \quad \text { for each } \theta \in S_{t}^{\prime},
\end{gathered}
$$

as

$$
\operatorname{dim}_{H}\left(K_{\alpha}^{+}\right)+\operatorname{dim}_{H}\left(K_{\beta}^{-}\right)-\operatorname{dim}_{H}(\Lambda)=\operatorname{dim}_{H}\left(S_{t}^{\prime}\right)=D(t)+1 .
$$

For the remaining proofs of assertions (3) to (5) we must modify some arguments from [2] slightly:

To (3) : We modify the proof of [2, Lemma 12.3.6] as follows. Since inequality (12.15) does no longer hold for all large $n$ 's, but still for infinitely many $n$ 's, several inequalities after the estimate (12.17) hold only for $r$ 's such that $m(\theta, r)$ defined in (12.12) satisfies (12.15) in place of $n$. Choose a null sequence $\left(r_{k}\right)_{k}$ of $r$ 's in the above manner. Then we obtain the last two inequalities in the proof of Lemma 12.3.6 where both limits superior are replaced by the limits inferior.

To (4) : We modify the proof of [2, Lemma 12.3.6] again. Let $t \in\left(\gamma_{\min }, \gamma_{c}^{-}\right)$such that $q(t)<0$. Then, for $\delta>0$ and $\chi(\omega) \in N_{t}^{\leqslant}$, there is a $r(\omega) \in \mathbb{N}$ such that for $n>r(\omega)$

$$
q(t) \cdot \sum_{k=0}^{n}\left(\Phi^{s}\left(\left(\sigma^{-}\right)^{k} \omega^{-}\right)-t\right)>-\delta n|q(t)| .
$$

Since we can obtain (12.17) from this estimate instead of (12.15), the proof is finished.

To (5) : Let $t \in\left(\gamma_{c}^{-}, \gamma_{\max }\right)$ such that $q(t)>0$. As in the proof of $(4)$, for $\delta>0$ and $\chi(\omega) \in N_{t}^{\geqslant}$ there is a $r(\omega) \in \mathbb{N}$ such that (49) holds for $n>r(\omega)$, and again we can obtain (12.17) from this estimate instead of (12.15).

Lemma 5. For $t \in\left(\gamma_{\min }, \gamma_{\max }\right)$ we have $\operatorname{dim}_{H}\left(S_{t}^{\prime}\right)=\operatorname{dim}_{H}\left(R_{t}^{\prime}\right)=\operatorname{dim}_{P}\left(S_{t}^{\prime}\right)=D(t)+1$. Furthermore, $\operatorname{dim}_{H}\left(N_{t}^{\leqslant}\right)=D(t)+1$ for $t \in\left(\gamma_{\min }, \gamma_{c}^{-}\right]$and $\operatorname{dim}_{P}\left(N_{t}^{\geqslant}\right)=D(t)+1$ for $t \in\left[\gamma_{c}^{-}, \gamma_{\max }\right)$. 
Proof of Lemma 5. This follows from Lemma 4 with [2, Theorem 2.1.5] and [4, Proposition 2.3].

Remark 11. Roughly speaking, each of the sets $S_{t}^{\prime}$ and $R_{t}^{\prime}$ is locally the product of a $D(t)$ dimensional subset of the local stable manifold and the complete local unstable manifold.

Proof of the Theorem 2. As $S_{t}^{\prime} \subseteq S_{t} \subseteq R_{t}^{\prime}$, we obtain $\operatorname{dim}_{H}\left(S_{t}\right)=D(t)+1$ for $t \in\left(\gamma_{\min }, \gamma_{\max }\right)$ from Lemma 5. For the remaining arguments observe also the monotonicity properties of $t \mapsto D(t)$ from Lemma 3 .

Let $t \in\left(\gamma_{\text {min }}, \gamma_{c}^{-}\right]$. Firstly, $D(t)+1=\lim _{t^{\prime} \nearrow_{t}} D\left(t^{\prime}\right)+1=\lim _{t^{\prime} \nearrow_{t}} \operatorname{dim}_{H}\left(S_{t^{\prime}}\right) \leqslant \operatorname{dim}_{H}\left(N_{t}\right)$, as $S_{t^{\prime}} \subseteq N_{t}$. Secondly, from Theorem 1 we have $N_{t} \subseteq\{\Gamma \leqslant t\} \subseteq R_{t}^{\prime} \cup N_{t}^{\leqslant}$. Thus, $\operatorname{dim}_{H}\left(N_{t}\right) \leqslant$ $\max \left\{\operatorname{dim}_{H}\left(R_{t}^{\prime}\right), \operatorname{dim}_{H}\left(N_{t}^{\leqslant}\right)\right\}=D(t)+1$, and it follows that $\operatorname{dim}_{H}\left(N_{t}\right)=D(t)+1$.

Now, let $t \in\left[\gamma_{c}^{-}, \gamma_{\max }\right)$. Firstly, $D(t)+1=\lim _{t^{\prime \prime} \backslash t} D(t)+1=\lim _{t^{\prime \prime} \backslash t} \operatorname{dim}_{H}\left(S_{t^{\prime \prime}}\right) \leqslant$ $\operatorname{dim}_{H}\left(\Theta \backslash N_{t}\right)$, as $S_{t^{\prime \prime}} \subseteq \Theta \backslash N_{t}$. Secondly, from Theorem 1 we have $\Theta \backslash N_{t} \subseteq N_{t}^{\geqslant}$. Thus, $\operatorname{dim}_{H}\left(\Theta \backslash N_{t}\right) \leqslant \operatorname{dim}_{P}\left(\Theta \backslash N_{t}\right) \leqslant \operatorname{dim}_{P}\left(N_{t}^{\geqslant}\right)=D(t)+1$, and it follows that $\operatorname{dim}_{H}\left(\Theta \backslash N_{t}\right)=$ $\operatorname{dim}_{P}\left(\Theta \backslash N_{t}\right)=D(t)+1$.

\subsection{Proof of Theorem 3 .}

Proof. To (2): Let $t \in\left[\gamma_{c}^{-}, \gamma_{\max }\right)$. For $t^{\prime} \in\left(t, \gamma_{\max }\right)$ and $\nu_{t^{\prime}} \in \mathcal{P}(\Theta)$ from Lemma 4 it follows $\nu_{t^{\prime}}\left(\bigcup_{k \in \mathbb{N}}\left\{\varphi_{t}>1 / k\right\}\right)=\nu_{t^{\prime}}\left(\Theta \backslash N_{t}\right) \geqslant \nu_{t^{\prime}}\left(S_{t^{\prime}}\right)=1$, whence there is a $k \in \mathbb{N}$ s.t. $\nu_{t^{\prime}}\left(\left\{\varphi_{t}>1 / k\right\}\right)>0$. Denoting the restriction of $\nu_{t^{\prime}}$ to this set by $\tilde{\nu}_{t^{\prime}}$ we obtain $\operatorname{dim}_{H}\left(\left\{\varphi_{t}>\right.\right.$ $1 / k\}) \geqslant \operatorname{dim}_{H}\left(\tilde{\nu}_{t^{\prime}}\right) \geqslant D\left(t^{\prime}\right)+1$ by [2, Theorem 2.1.5], as $d_{\tilde{\nu}_{t^{\prime}}}=d_{\nu_{t^{\prime}}}=D\left(t^{\prime}\right)+1$ holds $\tilde{\nu}_{t^{\prime}}$-a.e.. Therefore, $\operatorname{dim}_{H}\left(\left\{\varphi_{t}>1 / k\right\}\right) \geqslant \lim _{t^{\prime} \backslash t} D\left(t^{\prime}\right)+1=D(t)+1$, and it follows from the first product rule of [11, Theorem 3] that

$\operatorname{dim}_{H}\left(\mathcal{A}_{t}\right) \geqslant \operatorname{dim}_{H}\left(\left\{\varphi_{t}>1 / k\right\} \times[0,1 / k]\right) \geqslant \operatorname{dim}_{H}\left(\left\{\varphi_{t}>1 / k\right\}\right)+\operatorname{dim}_{H}([0,1 / k]) \geqslant D(t)+2$.

On the other hand, Theorem 2 in conjunction with the last product rule of [11, Theorem 3] implies

$$
\operatorname{dim}_{P}\left(\mathcal{A}_{t}\right) \leqslant \operatorname{dim}_{P}\left(\Theta \backslash N_{t} \times\left[0, M_{t}\right]\right) \leqslant \operatorname{dim}_{P}\left(\Theta \backslash N_{t}\right)+\operatorname{dim}_{P}\left(\left[0, M_{t}\right]\right)=D(t)+2,
$$

so that $\operatorname{dim}_{P}\left(\mathcal{A}_{t}\right)=\operatorname{dim}_{H}\left(\mathcal{A}_{t}\right)=D(t)+2$.

To (1): Let $t \in\left(\gamma_{\min }, \gamma_{c}^{-}\right]$. From $(2)$ and Theorem 2 it follows $\operatorname{dim}_{P}\left(\mathcal{A}_{t}\right) \geqslant \operatorname{dim}_{H}\left(\mathcal{A}_{t}\right) \geqslant$ $\operatorname{dim}_{H}\left(\mathcal{A}_{\gamma_{c}^{-}}\right)=D\left(\gamma_{c}^{-}\right)+1=3$, which finishes the proof.

\section{REFERENCES}

[1] L. Arnold. Random Dynamical Systems. Springer, 2 edition, 2003.

[2] L. Barreira. Dimension and recurrence in hyperbolic dynamics. Birkhäuser, 2008.

[3] R. Bowen. Equilibrium states and the ergodic theory of Anosov diffeomorphisms. Springer, 2 edition, 2007.

[4] K. Falconer. Techniques in Fractal Geometry. Wiley, 1997.

[5] G. Keller. A note on strange nonchaotic attractors. Fund. Math, 151:139-148, 1996.

[6] G. Keller. Equilibrium States in Ergodic Theory. Cambridge University Press, 1998.

[7] G. Keller, H. Jaffri, and R. Ramaswamy. Generalized synchronozation in chaotically driven systems. In preparation, 2012.

[8] W. Parry and M. Pollicott. Zeta functions and the periodic orbit structure of hyperbolic dynamics. Soc. Math. de France, 1990.

[9] J. Schmeling. Entropy preservation under markov coding. Journal of Statistical Physics, 104:799-815, 2001.

[10] R. Sturman and J. Stark. Semi-uniform ergodic theorems and applications to forced systems. Nonlinearity, 13:113, 2000.

[11] C. Tricot. Two definitions of fractional dimension. Math. Proc. Camb. Phil. Soc., 91:57-74, 1982. 
Department Mathematik, Universität Erlangen-Nürnberg, Cauerstr. 11, 91058 Erlangen, Germany

E-mail address: keller@mi.uni-erlangen.de

E-mail address: otani@mi.uni-erlangen.de 